\title{
HILBERT CUSP FORMS AND SPECIAL VALUES OF DIRICHLET SERIES OF RANKIN TYPE
}

\author{
by MIN HO LEE
}

(Received 31 July, 1996)

1. Introduction. Let $K$ be a totally real number field of degree $n$ over $\mathbb{Q}$, and let $\mathbf{c}$ be an integral ideal of a maximal order $\mathcal{O}_{K}$ of $K$. Given a nonnegative integer $j$ and a Hecke character on the group $A_{K}^{\times}$of ideles of $K$, let $\mathscr{S}(c, \psi)$ denote the space of Hilbert cusp forms of holomorphic type on $\mathscr{H}^{n}$ of weight $j$, level $c$ and character $\psi$, where $\mathscr{H}^{n}$ is the $n$-th power of the Poincaré upper half plane $\mathscr{H}$. Let $\mathbf{g}$ be an element of $\mathscr{S}_{1}(\mathrm{c}, \mathbf{1})$, where $\mathbf{1}$ is the trivial character. If $\mathbf{u} \in S_{k}(\mathfrak{c}, \psi)$, then the product gu is an element of $S_{k+\lambda}(\mathfrak{c}, \psi)$, and therefore we can consider the linear map $\Phi_{\mathrm{g}}: \mathscr{S}_{k}(\mathrm{c}, \psi) \rightarrow \mathscr{S}_{k+1}(\mathrm{c}, \psi)$ sending $\mathbf{u}$ to gu. Let $\Phi_{\mathrm{g}}^{*}: \mathscr{S}_{k+1}(c, \psi) \rightarrow \mathscr{S}_{k}(c, \psi)$ be the adjoint of the linear map $\Phi_{\mathrm{g}}$ with respect to the Petersson inner product.

In this paper we study the Fourier coefficients of $\Phi_{\mathrm{g}}^{*} \mathbf{f}$ associated to a Hilbert cusp form $\mathbf{f}$ of holomorphic type in $\mathscr{S}_{k+\lambda}(c, \psi)$. We define Dirichlet series of Rankin type associated to the Fourier coefficients of $\mathbf{g}$ and $\mathbf{f}$ and express the Fourier coefficients of $\Phi_{\mathbf{g}}^{*} \mathbf{f}$ in terms of special values of such Dirichlet series. Such a problem was treated by Kohnen [1] in the case of elliptic modular forms. In order to consider the Hilbert modular case we use holomorphic projection operators and Poincaré series of two variables used by Panchishkin [3] to prove the algebraicity of a certain expression.

2. Hilbert automorphic forms. In this section we review Hilbert automorphic forms using the language of adeles (see e.g. [2], [3] for details). Let $K$ be a totally real number field of degree $n$ over $\mathbb{Q}, \mathbb{A}_{K}$ its ring of adeles, $\mathcal{O}_{K}$ a maximal order, and $I_{K}$ the group of fractional ideals of $K$. Let $G$ be an algebraic group over $\mathbb{Q}$ such that $G(\mathbb{Q})=G L(2, K)$. If $\hat{\mathcal{O}}_{K}$ is the profinite completion of $\hat{O}_{K}$, then $\hat{K} \cong \hat{\mathcal{O}}_{K} \times_{\mathbb{Z}} \mathbb{Q}$ is the subring of $\mathbb{A}_{K}$ of finite adeles and we have $\mathbb{A}_{K}=K_{x} \times \hat{K}$, where $K_{\infty}$ is the subring of $\mathbb{A}_{K}$ of adeles at infinity. If $\mathbb{A}$ is the ring of adeles of $\mathbb{Q}$, then we have

$$
G(\mathbb{A})=G L\left(2, \mathbb{A}_{K}\right)=G_{x} \times G L(2, \hat{K}),
$$

where $G_{x}=G L\left(2, K_{x}\right)$. Since $K$ is totally real we can identify $G_{x}=G L\left(2, K_{x}\right)$ with $G L(2, \mathbb{R})^{n}$. Under this identification, let $G_{x}^{+}$be the subgroup of $G L(2, \mathbb{R})^{n}$ consisting of elements $\alpha=\left(\alpha_{1}, \ldots, \alpha_{n}\right)$ with

$$
\alpha_{j}=\left(\begin{array}{ll}
a_{j} & b_{j} \\
c_{j} & d_{j}
\end{array}\right) \in G L(2, \mathbb{R}), \quad \operatorname{det} \alpha_{j}>0
$$

for all $j=1, \ldots, n$. Let $\mathscr{H}^{n}$ be the $n$-fold power of the Poincaré upper half plane

$$
\mathscr{H}=\{z \in \mathbb{C} \mid \operatorname{Im} z>0\} .
$$

Then each element $\alpha \in G_{x}^{+}$acts on $\mathscr{H}^{\prime \prime}$ by

$$
\alpha \cdot z=\left(\alpha_{1} z_{1}, \ldots, \alpha_{n} z_{n}\right) \in \mathscr{H}^{n}
$$

Glasgow Math. J. 40 (1998) 71-77. 
for $z=\left(z_{1}, \ldots, z_{n}\right) \in \mathscr{H}^{n}$, where

$$
\alpha_{j} z_{j}=\left(a_{j} z_{j}+b_{j}\right)\left(c_{j} z_{j}+d_{j}\right)^{-1} \in \mathscr{H}, \quad j=1, \ldots, n .
$$

For $k \in \mathbb{Z}$ and $z=\left(z_{1}, \ldots, z_{n}\right) \in \mathscr{H}^{n}$, we set

$$
\mathbf{e}(z)=e^{2 \pi i\left(z_{1}+\ldots+z_{n}\right)}, \quad \mathcal{N}(z)^{k}=z_{1}^{k} \ldots z_{n}^{k}
$$

Given a function $f: \mathscr{H}^{n} \rightarrow \mathbb{C}$ and $\alpha \in G_{\infty}^{+}$we define the function $\left.f\right|_{k} \alpha: \mathscr{H}^{n} \rightarrow \mathbb{C}$ by

$$
\left(\left.f\right|_{k} \alpha\right)(z)=\mathcal{N}(c z+d)^{-k} f(\alpha z) \mathcal{N}(\operatorname{det} \alpha)^{k / 2}
$$

for all $z \in \mathscr{K}^{n}$, where $c z+d=\left(c_{1} z_{1}+d_{1}, \ldots, c_{n} z_{n}+d_{n}\right) \in \mathscr{H}^{n}$.

Let $c \subset \mathcal{O}_{K}$ be an integral ideal, and for each place $\mathfrak{p}$ of $K$ let $\mathfrak{c}_{\mathfrak{p}}=\mathfrak{c} \mathcal{O}_{\mathfrak{p}}$ be its $\mathfrak{p}$-part. Let $D$ be the different of $K$, and let $\delta_{\mathfrak{p}}=\delta \sigma_{\mathfrak{p}}$ be the associated local different at $\mathfrak{p}$. We define the open subgroup $W=W(\mathrm{c})$ of $G(\mathbb{A})$ by

$$
W=G_{\infty}^{+} \times \prod_{\mathfrak{p}} W(\mathfrak{p})
$$

where

$$
W(\mathfrak{p})=\left\{\left(\begin{array}{ll}
a & b \\
c & d
\end{array}\right) \in G L\left(2, K_{\mathfrak{p}}\right) \mid b \in \mathfrak{D}_{\mathfrak{p}}^{-1}, c \in \mathfrak{D}_{\mathfrak{p}} \mathfrak{c}_{\mathfrak{p}}, a, d \in \mathcal{O}_{\mathfrak{p}}, a d-b c \in \mathscr{O}_{\mathfrak{v}}^{\times}\right\}
$$

If $K_{+}^{\times}$denotes the multiplicative group of all totally positive elements of $K$, then the quotient $I_{K} / K_{+}^{\times}$is the ideal class group of $K$. Let $h=\left|I_{K} / K_{+}^{\times}\right|$be the class number of $K$, and let $\left\{t_{1}, \ldots, t_{h}\right\}$ be the set of ideles such that their images $\tilde{t}_{v}$ in $\tilde{O}_{K}$ form a complete system of representatives for $I_{K} / K_{+}^{\times}$and

$$
\left(t_{v}\right)_{\infty}=1, \quad \tilde{t}_{v}+\mathrm{m}_{0}=\mathcal{O}_{K}, \quad \mathrm{~m}_{0}=\prod_{\mathfrak{q} \in S_{K}} \mathfrak{q}
$$

for $1 \leq v \leq h$, where $S$ is a finite set of primes and $S_{K}$ is the set of primes $\mathfrak{p}$ dividing each element of $S$. Then we have

$$
G(\mathbb{A})=\bigcup_{v=1}^{h} G(\mathbb{Q}) x_{v} W=\bigcup_{v=1}^{h} G(\mathbb{Q}) x_{v}^{-i} W
$$

where $x_{v}=\left(\begin{array}{ll}1 & 0 \\ 0 & t_{v}\end{array}\right)$ and $i$ denotes the involution of $2 \times 2$ matrices given by

$$
\left(\begin{array}{ll}
a & b \\
c & d
\end{array}\right)^{i}=\left(\begin{array}{cc}
d & -b \\
-c & a
\end{array}\right)
$$

Definition 2.1. Let $c \subset \mathcal{O}_{\mathrm{K}}$ be an integral ideal as above, and let $\psi: A_{K}^{\times} \rightarrow \mathbb{C}^{\times}$be a Hecke character. A Hilbert automorphic form of weight $k$, level $c$, and character $\psi$ is a function $\mathbf{f}: G(\mathbb{A}) \rightarrow \mathbb{C}$ satisfying the following conditions:

(i) $\mathbf{f}(s \alpha x)=\psi(s) \mathbf{f}(x)$ for all $x \in G(\mathbb{A}), s \in \mathbb{A}_{K}^{\times}$and $\alpha \in G(\mathbb{Q})$;

(ii) $\mathbf{f}(x w)=\psi\left(w^{\prime}\right) \mathbf{f}(x)$ for $w \in W$ with $w_{\infty}=1$;

(iii) $\mathbf{f}(x w(\theta))=\mathbf{f}(x) e^{-i k\left(\theta_{1}+\ldots+\theta_{n}\right)}$ for all $x \in G(\mathbb{A})$ and

$$
w(\theta)=\left(w_{1}\left(\theta_{1}\right), \ldots, w_{n}\left(\theta_{n}\right)\right) \in W
$$


where

$$
w_{j}\left(\theta_{j}\right)=\left(\begin{array}{cc}
\cos \theta_{j} & -\sin \theta_{j} \\
\sin \theta_{j} & \cos \theta_{j}
\end{array}\right), \quad j=1, \ldots, n .
$$

A Hilbert cusp form is a Hilbert automorphic form satisfying the additional condition that

$$
\int_{\mathbb{A}_{K} / K} \mathbf{f}\left(\left(\begin{array}{ll}
1 & t \\
0 & 1
\end{array}\right) g\right) d t=0
$$

for all $g \in G(\mathbb{A})$.

Definition 2.2. A Hilbert automorphic (resp. cusp) form of holomorphic type is a Hilbert automorphic (resp. cusp) form such that for any $x \in G(\mathbb{A})$ with $x_{\infty}=1$ there exists a holomorphic function $g_{x}: \mathscr{H}^{n} \rightarrow \mathbb{C}$ with $\mathbf{f}(x y)=\left(\left.g_{x}\right|_{k} y\right)(\mathbf{i})$ for all $y \in G_{\infty}^{+}$, where $\mathbf{i}=(i, \ldots, i) \in \mathscr{H}^{n}$. We denote by $\mathcal{M}_{k}(\mathrm{c}, \psi)$ (resp. $\left.\mathscr{S}_{k}(\mathrm{c}, \psi)\right)$ the space of such Hilbert automorphic (resp. cusp) forms of weight $k$, level $c$ and character $\psi$ of holomorphic type.

For $\mathbf{f} \in \mathcal{M}_{k}(\mathfrak{c}, \psi)$ and $1 \leq v \leq h$, set $f_{v}=g x_{v}^{-i}$, and let

$$
\begin{aligned}
\Gamma_{v} & =\Gamma_{v}(c)=x_{v} W x_{v}^{-1} \cap G(\mathbb{Q}) \\
& =\left\{\left(\begin{array}{ll}
a & b \\
c & d
\end{array}\right) \in G^{+}(\mathbb{Q}) \mid b \in \tilde{t}_{v}^{-1} b^{-1}, c \in \tilde{t}_{v} b c, a, d \in \mathcal{O}_{K}, a d-b c \in \mathcal{O}_{K}^{\times}\right\} .
\end{aligned}
$$

Then $f_{v}$ is a Hilbert modular form of weight $k$ and character $\psi$ for the congruence subgroup $\Gamma_{v}$. Thus it satisfies $\left.f_{v}\right|_{k} \gamma=\psi(\gamma) f_{v}$, and has a Fourier expansion of the form

$$
f_{v}(z)=\sum_{\xi} a_{v}(\xi) \mathbf{e}(\xi z)
$$

where $0<\xi \in \tilde{t}_{v}$ or $\xi=0$. If $\mathcal{M}_{k}\left(\Gamma_{v}, \psi\right)$ denotes the space of Hilbert modular forms of weight $k$ and character $\psi$ for $\Gamma_{v}$, then the map $\mathbf{f} \mapsto\left(f_{1}, \ldots, f_{h}\right)$ determines a canonical isomorphism

$$
\mathcal{M}_{k}(\mathfrak{c}, \psi) \cong \bigoplus_{v=1}^{h} \mathcal{M}_{k}\left(\Gamma_{\nu}, \psi\right)
$$

We shall identify $\mathcal{M}_{k}(\mathfrak{c}, \psi)$ with $\bigoplus_{v=1}^{h} \mathcal{M}_{k}\left(\Gamma_{v}, \psi\right)$ so that $\mathbf{f}=\left(f_{1}, \ldots, f_{h}\right)$. Similarly, we have

$$
\mathscr{S}_{k}(\mathfrak{c}, \psi)=\bigoplus_{v=1}^{h} \mathscr{S}_{k}\left(\Gamma_{v}, \psi\right), \quad \mathscr{S}_{k}\left(\Gamma_{v}, \psi\right)=\mathscr{S}_{k}(\mathfrak{c}, \psi) \cap \mathcal{M}_{k}\left(\Gamma_{v}, \psi\right)
$$

If $\mathbf{f}=\left(f_{1}, \ldots, f_{h}\right) \in \mathscr{S}_{k}(\mathfrak{c}, \psi)$ and $\mathbf{g}=\left(g_{1}, \ldots, g_{h}\right) \in \mathcal{M}_{k}(\mathfrak{c}, \psi)$, then the Petersson inner product is defined by

$$
\langle\mathbf{f}, \mathbf{g}\rangle=\sum_{v=1}^{h} \int_{\Gamma_{v} \backslash \mathscr{R}^{n}} \overline{f_{v}(z)} g_{v}(z) \mathcal{N}(y)^{k} d \mu(z),
$$

where $z=\left(z_{1}, \ldots, z_{n}\right), z_{j}=x_{j}+i y_{j}(1 \leq j \leq n)$, and

is a $G_{\infty}^{+}$-invariant measure on $\mathscr{H}^{n}$.

$$
d \mu(z)=\prod_{j=1}^{n} y_{j}^{-2} d x_{j} d y_{j}
$$


Definition 2.3. $A C^{\infty}$ Hilbert automorphic form of weight $k$, level $\mathfrak{c}$, and character $\psi$ is a function $F: G(\mathbb{A}) \rightarrow \mathbb{C}$ satisfying the following conditions:

(i) $F$ satisfies the conditions (i), (ii) and (iii) in Definition 2.1.

(ii) For each $x \in G(\mathbb{A})$ with $x_{\infty}=1$ there exists a $C^{\infty}$ function $g_{x}: \mathscr{H}^{n} \rightarrow \mathbb{C}$ with $F(x y)=\left(\left.g_{x}\right|_{k} y\right)(i)$ for all $y \in G_{\infty}$.

We shall denote by $\tilde{M}_{k}(\mathfrak{c}, \psi)$ the space of all such $C^{\infty}$ Hilbert automorphic forms.

For $1 \leq v \leq h$, let $F_{v}=g_{x_{v}^{-i}}$. Then $F_{v}$ is a $C^{\infty}$ Hilbert modular form on $\mathscr{H}^{n}$ of weight $k$ and character $\psi$ relative to the congruence subgroup $\Gamma_{v}$. In particular, it satisfies $\left(\left.F_{v}\right|_{k} \gamma\right)=\psi(\gamma) F_{v}$ for each $\gamma \in \Gamma_{v}$ and has a Fourier expansion of the form

$$
F_{v}(z)=\sum_{\xi \in \tilde{I}_{v}} a_{v}(\xi, y) \mathbf{e}(\xi x)
$$

where the maps $y \mapsto a_{v}(\xi, y)$ are $C^{\infty}$ functions on

$$
\left(\mathbb{R}_{+}\right)^{n}=\left\{y=\left(y_{1}, \ldots, y_{n}\right) \mid y_{j}>0 \text { for all } j\right\} .
$$

If $\mathscr{M}_{k}\left(\Gamma_{v}, \psi\right)$ denotes the space of all $C^{\infty}$ Hilbert modular forms of weight $k$ and character $\psi$ for $\Gamma_{v}$, then we have

$$
\tilde{M}_{k}(\mathfrak{c}, \psi) \cong \bigoplus_{v=1}^{h} \tilde{M}_{k}\left(\Gamma_{v}, \psi\right)
$$

As in the case of $\mathcal{M}_{k}(\mathfrak{c}, \psi)$ and $\mathscr{S}_{k}(\mathfrak{c}, \psi)$, we shall identity $F \in \widetilde{M}_{k}(\mathfrak{c}, \psi)$ with its image $\left(F_{1}, \ldots, F_{h}\right)$ in $\bigoplus_{v=1}^{h} \tilde{M}_{k}\left(\Gamma_{v}, \psi\right)$ under this isomorphism. The Petersson inner product in (1) can be extended to elements $\mathbf{f} \in \mathscr{S}_{k}(\mathfrak{c}, \psi)$ and $F \in \mathscr{M}_{k}(\mathfrak{c}, \psi)$.

THEOREM 2.4. Let $F=\left(F_{1}, \ldots, F_{h}\right) \in \widetilde{M}_{k}(\mathfrak{c}, \psi)$ be a $C^{\infty}$ Hilbert automorphic form of moderate growth such that for each $v$ the Fourier expansion

$$
F_{v}(z)=\sum_{\xi \in \tilde{t}_{v}} a_{v}(\xi, y) \mathbf{e}(\xi x)
$$

contains only terms with totally positive $\xi \in \tilde{t}_{v}$. For $1 \leq v \leq h$, we set

$$
a_{\nu}(\xi)=\frac{(4 \pi)^{n(k-1)}(\mathcal{N} \xi)^{k-1}}{\Gamma(k-1)^{n}} \int_{\left(\mathbb{R}_{+}\right)^{n}} a_{\nu}(\xi, y) \mathbf{e}(i \xi y) y^{k-2} d y,
$$

where $\Gamma(k-1)$ is the value of the gamma function

$$
\Gamma(s)=\int_{0}^{\infty} e^{-y} y^{s-1} d y
$$

at $s=k-1$. If $F^{H}=\left(F_{1}^{H}, \ldots, F_{h}^{H}\right)$ with

$$
F_{v}^{H}(z)=\sum_{0<\xi \in i_{v}} a_{v}(\xi) \mathbf{e}(\xi z)
$$

for each $v$, then $F^{H}$ is a Hilbert automorphic form of holomorphic type in $M_{k}(\mathfrak{c}, \psi)$ and

$$
\langle g, F\rangle=\left\langle g, F^{H}\right\rangle
$$

for all $g \in \mathscr{S}_{k}(\mathrm{c}, \psi)$.

Proof. See Proposition 4.7 in [3, Chapter 4]. 
3. Fourier coefficients. Let $\mathbf{g}=\left(g_{1}, \ldots, g_{h}\right)$ be an element of $\mathscr{S}_{l}(\mathbf{c})=\mathscr{S}_{l}(\mathrm{c}, \mathbf{1})=$ $\bigoplus_{v=1} \mathscr{S}_{\lambda}\left(\Gamma_{v}, \mathbf{1}\right)$, where 1 denotes the trivial character, and let $\mathbf{u}=\left(u_{1}, \ldots, u_{h}\right) \in \mathscr{S}_{k}(\mathrm{c}, \psi)=$ $\bigoplus_{v=1}^{\natural} \mathscr{S}_{k}\left(\Gamma_{v}, \psi\right)$. Then the product gu is an element of $\mathscr{S}_{k+1}(\mathrm{c}, \psi)$. Thus we can consider the linear map $\Phi_{\mathrm{g}}: \mathscr{S}_{k}(\mathrm{c}, \psi) \rightarrow \mathscr{S}_{k+\lambda}(\mathrm{c}, \psi)$ sending $\mathbf{u} \in \mathscr{S}_{k}(\mathrm{c}, \psi)$ to gu $\in \mathscr{S}_{k+\lambda}(\mathrm{c}, \psi)$. Let

$$
\Phi_{\mathrm{g}}^{*}: \mathscr{S}_{k+1}(\mathfrak{c}, \psi) \rightarrow \mathscr{S}_{k}(\mathrm{c}, \psi)
$$

be the adjoint of the linear map $\Phi_{\mathrm{g}}$ with respect to the Petersson inner product. For $1 \leq v \leq h$ let $P_{k, v}^{\psi}$ be the Poincaré series of two variables given by

$$
P_{k, v}^{\psi}(z, w, s)=\sum_{\gamma \in \Gamma_{v}} \psi(\gamma) J(\gamma, z)^{-k}|J(\gamma, z)|^{-2 s}(\gamma s+s)^{k}|\gamma s+s|^{-2 s},
$$

for $z, w \in \mathscr{H}^{\prime \prime}$, where $J(\gamma, z)=\operatorname{det} \gamma^{-1 / 2}(c z+d)$ for $\gamma=\left(\begin{array}{ll}a & b \\ c & d\end{array}\right) \in \Gamma_{\nu}$. The series in (4) converges absolutely and uniformly on any compact subset of $\mathscr{K}^{n} \times \mathscr{K}^{n}$ for $k+\operatorname{Re}(2 s)>2$ (see [3, p. 139]).

Lemma 3.1. If $g_{v} \in \mathscr{S}_{(}\left(\Gamma_{v}, 1\right)$ and $f_{v} \in \mathscr{S}_{k+l}\left(\Gamma_{v}, \psi\right)$, then the function

$$
f_{v}(z) \overline{g_{v}(z)} \mathcal{N}(\operatorname{Im} z)^{\prime}
$$

is a $C^{\infty}$ Hilbert modular form in $\tilde{\mathcal{M}}_{k}\left(\Gamma_{v}, \psi\right)$ of moderate growth.

Proof. For each $\gamma \in \Gamma_{v}$ we have

$$
f_{v}(\gamma z)=\psi(\gamma) J(\gamma, z)^{k+l} f_{v}(z), \quad \overline{g_{v}(\gamma z)}=\overline{J(\gamma, z)^{\prime}} \overline{g_{v}(z)}
$$

Since $\mathcal{N}(\operatorname{Im} \gamma z)^{\prime}=|J(\gamma, z)|^{-2 \prime} \mathcal{N}(\operatorname{Im} z)^{\prime}$, it follows that

$$
f_{v}(\gamma z) \overline{g_{v}(\gamma z)} \mathcal{N}(\operatorname{Im} \gamma z)^{\prime}=\psi(\gamma) J(\gamma, z)^{k} f_{v}(z) \overline{g_{v}(z)} \mathcal{N}(\operatorname{Im} z)^{\prime} .
$$

Furthermore, from the cusp conditions for $f_{v}$ and $g_{v}$ it follows that $f(z) \overline{g(z)} \mathcal{N}(\operatorname{Im} z)^{l}$ is of moderate growth.

Proposition 3.2. Let $\mathbf{g} \in \mathscr{S}_{l}(\mathrm{c})=\mathscr{S}_{l}(\mathrm{c}, \mathbf{1}), \mathbf{f} \in \mathscr{S}_{k+1}(c, \psi)$, and let $F^{H} \in \mathscr{S}_{k}(\mathrm{c}, \psi)$ be the Hilbert automorphic form of holomorphic type associated to the $C^{\times}$Hilbert automorphic form

$$
F(z)=\mathbf{f}(z) \overline{\mathbf{g}(z)} \mathcal{N}(\operatorname{Im} z)^{\prime}
$$

Then we have $\Phi_{\mathrm{g}}^{*}(\mathbf{f})=F^{H}$.

Proof. Let $\mathbf{f}=\left(f_{1}, \ldots, f_{h}\right) \in \mathscr{S}_{k+1}(\mathrm{c}, \psi), \mathbf{g}=\left(g_{1}, \ldots, g_{h}\right) \in \mathscr{S}_{l}(\mathrm{c})$, and let $P_{k, v}^{\psi}$ be the Poincaré series given by (4). Then for $1 \leq v \leq h$ we have

$$
\begin{aligned}
\left\langle P_{k, v}^{\psi}(-\bar{z}, w, s),\left(\Phi_{\mathrm{g}}^{*} f_{v}\right)(w)\right\rangle & =\left\langle\Phi_{\mathrm{g}} P_{k, v}^{\psi}(-\bar{z}, w, s), f_{v}(w)\right\rangle \\
& =\left\langle g_{v}(w) P_{k, v}^{\psi}(-\bar{z}, w, s), f_{v}(w)\right\rangle \\
& =\int_{D_{v}} \overline{g_{v}(w) P_{k, v}^{\psi}(-\bar{z}, w, s)} f_{v}(w) \mathcal{N}(v)^{k+l} d \mu(w) \\
& =\int_{D_{v}} \overline{P_{k, v}^{\psi}(-\bar{z}, w, s)}\left(f_{v}(w) \overline{g_{v}(w)} \mathcal{N}(v)^{l}\right) \mathcal{N}(v)^{k} d \mu(w) \\
& =\left\langle P_{k, v}^{\psi}(-\bar{z}, w, s), F_{v}(w)\right\rangle,
\end{aligned}
$$



where $w=\left(w_{1}, \ldots, w_{n}\right), w_{j}=u_{j}+i v_{j}$ and $d \mu(w)=\prod_{j=1}^{n} v_{j}^{-2} d u_{j} d v_{j}$. If $F^{H}=\left(F_{1}^{H}, \ldots, F_{h}^{H}\right)$,
then by (3) we obtain

$$
\left\langle P_{k, v}^{\psi}(-\bar{z}, w, s),\left(\Phi_{\mathrm{g}}^{*} f_{v}\right)(w)\right\rangle=\left\langle P_{k, v}^{\psi}(-\bar{z}, w, s), F_{v}^{H}(w)\right\rangle .
$$

Thus it follows from $[3$, p. 139] that

for $1 \leq v \leq h$, where

$$
c(k, s) \Phi_{\mathbf{g}}^{*} f_{v}(z)=c(k, s) F_{v}^{H}(z)
$$

Hence we have

$$
c(k, s)=2^{n(2-s-k)} i^{-n k} \pi^{n}(k+s-1)^{-n} .
$$

$$
\Phi_{\mathrm{g}}^{*} f_{v}(z)=F_{v}^{\prime \prime}(z)
$$

for all $v$, and therefore the proposition follows.

By Proposition 3.2, given $\mathbf{f} \in \mathscr{S}_{k+l}(c, \psi)$, we have

$$
\Phi_{\mathbf{g}}^{*} \mathbf{f}=F^{H}=\left(F_{1}^{H}, \ldots, F_{h}^{\prime \prime}\right) \in \mathscr{Y}_{k}(\mathrm{c}, \psi),
$$

and each $F_{v}^{H} \in \mathscr{S}_{k}\left(\Gamma_{v}, \psi\right)$ has a Fourier expansion of the form

$$
F_{v}^{H}(z)=\sum_{0<\xi \in i_{v}} a_{v}(\xi) \mathbf{e}(\xi z)
$$

Let $\mathbf{f}=\left(f_{1}, \ldots, f_{h}\right)$ and $\mathbf{g}=\left(g_{1}, \ldots, g_{h}\right)$ be as in Proposition 3.2 with

$$
f_{\nu}(z)=\sum_{0<\xi \in \tilde{i}_{v}} A_{v}(\xi) \mathbf{e}(\xi x), \quad g_{v}(z)=\sum_{0<\xi \in \tilde{i}_{v}} B_{v}(\xi) \mathbf{e}(\xi x)
$$

for $1 \leq v \leq h$. Then for $\xi \in \tilde{t}_{v}$ we define the Dirichlet series $L_{v}(\mathbf{f}, \mathbf{g}, \xi, s)$ of Rankin type by

$$
L_{v}(\mathbf{f}, \mathbf{g}, \xi, s)=\sum_{0<\eta \in \tilde{i}_{v}} \frac{A_{v}(\xi+\eta) \overline{B_{v}(\eta)}}{\mathcal{N}(\xi+\eta)^{s}}
$$

THEOREM 3.3. Let $\mathbf{f} \in \mathscr{S}_{k+1}(\mathrm{c}, \psi)$ and $\mathbf{g} \in \mathscr{S}_{1}(\mathrm{c})$ be as above, and let $a_{v}(\xi)$ be the Fourier coefficients of the component $F_{v}^{\prime \prime}$ of $\Phi_{\mathbf{g}}^{*} \mathbf{f}$ given in (5). Then we have

$$
a_{v}(\xi)=\frac{\Gamma(k+l-1)^{n}(\mathcal{N} \xi)^{k-1}}{(4 \pi)^{n /} \Gamma(k-1)^{n}} L_{v}(\mathbf{f}, \mathbf{g}, \xi, k+l-1)
$$

for $1 \leq v \leq h$ and $0<\xi \in \tilde{t}_{\gamma}$.

Proof. By (2) we have

$$
a_{v}(\xi)=C \int_{\left(\mathbb{\mathbb { B } _ { + } ) ^ { \prime \prime }}\right.} a_{v}(\xi, y) \mathbf{e}(i \xi y) y^{k-2} d y
$$


where

$$
C=\frac{(4 \pi)^{n(k-1)}(\mathcal{N} \xi)^{k-1}}{\Gamma(k-1)^{n}}
$$

Since $F_{v}(z)=f_{v}(z) \overline{g_{v}(z)} \mathcal{N}(\operatorname{Im} z)^{\prime}$, if $A_{v}$ and $B_{v}$ are as in (6), we have

$$
\begin{aligned}
F_{v}(z) & =\sum_{v} a_{v}(\xi, y) \mathbf{e}(\xi x) \\
& =\sum_{\xi, \eta} A_{v}(\mu) \overline{B_{v}(\eta)} \mathbf{e}((\mu-\eta) x) \mathbf{e}(i(\mu+\eta) y) \mathcal{N} y^{\prime} .
\end{aligned}
$$

Using $\xi=\mu-\eta$ or $\mu=\xi+\eta$ we obtain

$$
a_{v}(\xi, y)=\sum_{\eta} A_{v}(\xi+\eta) \overline{B_{v}(\eta)} \mathbf{e}(i(\xi+2 \eta) y) \mathcal{N} y^{\prime} .
$$

Hence it follows that

$$
\begin{aligned}
a_{v}(\xi) & =C \int_{\left(\mathbb{R}_{+}\right)^{\prime \prime}} \sum_{\eta} A_{v}(\xi+\eta) \overline{B_{v}(\eta)} \mathbf{e}(2 i(\xi+\eta) y) \mathcal{N} y^{k+1-2} d y \\
& =C \sum_{\eta} A_{v}(\xi+\eta) \overline{B_{v}(\eta)} \int_{\left(\mathbb{R}_{+}\right)^{n}} e^{-4 \pi \sum_{j^{-1}}\left(\xi_{j}+\eta_{i}\right) y_{j} \mathcal{N} y^{k+1-2} d y} \\
& =C \sum_{\eta} A_{v}(\xi+\eta) \overline{B_{v}(\eta)} \prod_{j=1}^{n} \int_{0}^{\infty} e^{-4 \pi\left(\xi_{0}+\eta_{j}\right) y_{j} y_{j}^{k+l-2}} d y_{j}
\end{aligned}
$$

Thus, using $v_{j}=4 \pi\left(\xi_{j}+\eta_{j}\right) y_{j}$ for $1 \leq j \leq n$, we have

$$
\begin{aligned}
a_{v}(\xi) & =C \sum_{\eta} A_{v}(\xi+\eta) \overline{B_{v}(\eta)} \prod_{j=1}^{n}\left(\int_{0}^{\infty} e^{-v_{i}} v_{j}^{k+1-2} d v_{j}\right) \prod_{j=1}^{n}\left(4 \pi\left(\xi_{j}+\eta_{j}\right)\right)^{-k-1+1} \\
& =C \sum_{\eta} A_{v}(\xi+\eta) \overline{B_{v}(\eta)} \Gamma(k+l-1)^{n}(4 \pi)^{n(-k-l+1)} \mathcal{N}(\xi+\eta)^{-k-l+1}
\end{aligned}
$$

Hence the theorem follows from this and the relations (7) and (8).

\section{REFERENCES}

1. W. Kohnen, Cusp forms and special values of certain Dirichlet series, Math. Z. 207 (1991), $657-660$.

2. Y. Manin, Non-archimedean integration and Jacquet-Langlands p-adic L-functions, Russian Math. Surveys 31 (1976), 5-57.

3. A. Panchishkin, Non-archimedean L-functions of Siegel and Hilbert modular forms, Springer-Verlag, New York, 1991.

Department of Mathematics

UNIVERSITY OF NORTHERN IOWA

Cedar Falls, Iowa 50614

U.S.A.

e-mail: lee@math.uni.edu 